\title{
Radiation Characteristics of 3D Resonant Cavity Antenna with Grid-Oscillator Integrated Inside
}

\author{
L. A. Haralambiev ${ }^{1}$ and H. D. Hristov ${ }^{2}$ \\ ${ }^{1}$ Department of Radio Engineering, Technical University of Varna, 9010 Varna, Bulgaria \\ ${ }^{2}$ Departamento de Electrónica, Universidad Técnica Federico Santa María, Avenida España 1680, Valparaíso, Chile
}

Correspondence should be addressed to H. D. Hristov; hristo.hristov@usm.cl

Received 17 July 2013; Accepted 1 December 2013; Published 16 January 2014

Academic Editor: Ahmad Safaai-Jazi

Copyright (c) 2014 L. A. Haralambiev and H. D. Hristov. This is an open access article distributed under the Creative Commons Attribution License, which permits unrestricted use, distribution, and reproduction in any medium, provided the original work is properly cited.

\begin{abstract}
A three-dimensional (3D) rectangular cavity antenna with an aperture size of $80 \mathrm{~mm} \times 80 \mathrm{~mm}$ and a length of $16 \mathrm{~mm}$, integrated with a four-MESFET transistor grid-oscillator, is designed and studied experimentally. It is found that the use of 3D antenna resonant cavity in case of small or medium gain microwave active cavity antenna leads to effective and stable power combining and radiation. The lack of lateral cavity diffraction and radiation helps in producing a directive gain of about $17 \mathrm{~dB}$ and radiation aperture efficiency bigger than $75 \%$ at a resonance frequency of $8.62 \mathrm{GHz}$. Good DC to RF oscillator efficiency of $26 \%$, effective isotropic radiated power (EIRP) of $5.2 \mathrm{~W}$, and SSB spectral power density of $-82 \mathrm{dBc} / \mathrm{Hz}$ are found from the measured data. The $3 \mathrm{D}$ antenna cavity serves also as a strong metal container for the solid-state oscillator circuitry.
\end{abstract}

\section{Introduction}

The miniaturization in antennas is of great significance for the modern mobile and portable aircraft and spacecraft wireless electronic systems. However, in addition to the structural, technological, power, and other limitation factors valid for all electronic devices, the miniaturization in antennas is inherently restricted by the space electromagnetic radiation mechanism.

Great antenna size reduction is achieved by using the resonant field phenomenon. The resonant antenna is usually commensurable in size with the design wavelength. If the outer resonant antenna surface is excited in resonance, the antenna is termed exoresonant. Examples of such antennas are the resonant dipoles or monopoles, loops, and slots. By analogy, if some antenna comprises a cavity tuned in resonance it is named endoresonant or simply cavity antenna (CA).

Depending on the configuration and inside field mode structure the passive single-cavity antennas are divided into two basic classes: closed or 3D cavity antennas [1-5] and open or beam-waveguide (Fabry-Perot) cavity antennas [613]. The classic optical or quasi-optical Fabry-Perot resonator is a laterally open, one-dimensional (1D) resonant cavity with a big transverse dimension $A \gg \lambda_{0}$ and length $L \approx$ $n \lambda_{0} / 2$, where $\lambda_{0}$ is the resonance cavity design frequency and $n \geq 1$ is an integer number. Frequently in practice, the microwave Fabry-Perot or 1D cavity antennas do not obey the above quasi-optical size condition. The above referenced 1D cavity antennas are based on fundamental-mode cavity resonators with a smaller transverse size of about several wavelengths and a length near to half the wavelength. As a rule, this compromise is on account of the antenna radiation properties. Most of the 1D passive cavity antennas have relatively low aperture radiation efficiency. For example, the radiation efficiency is $15-53 \%$ in [8], $41 \%$ in [9], $43 \%$ in [10], and $30-40 \%$ in [11]. An exception is claimed in [7], where a maximum efficiency of $75 \%$ is predicted.

Both 3D and 1D cavity antennas have partially reflecting surfaces (PRS) named also electromagnetic band gap (EBG) structures that serve as antenna radiation apertures. An arrangement of a 3D passage cavity and $3 \mathrm{D}$ or $1 \mathrm{D}$ radiating cavity forms a double-cavity antenna [3].

Due to the cavity-field resonance the passive $3 \mathrm{D}$ and $1 \mathrm{D}$ antennas are narrowband, with a typical frequency bandwidth from about 1 to $3 \%$ and a gain of $15-20 \mathrm{~dB}$. For these 
bandwidth and gain ranges the cavity antennas are much smaller in length, volume, and weight compared to the equal in aperture nonresonant antennas.

Along with the development of compact high-power communication, radar, and radionavigation equipment the need of a large-scale integration of electronic devices like microwave/mm-wave detectors, amplifiers, oscillators, phase-shifters, and so forth with the antenna structures is taking place, and thus, the antennas become active devices. The active antennas belong to the so-called functionally small antennas, a term that applies where additional performance is being added without increasing the antenna size [14].

An efficient power combining in a free space or in a cavity antenna volume is possible using a distributed (array) oscillator approach. The oscillating solid-state devices (Gunn or IMPATT diodes, MESFET or HEMT transistors, etc.) are mounted in a grid array. The grid-oscillator arrays act simultaneously as power combiners and antennas.

The open cavity antenna-oscillator (CAO) approach for combining the output powers of multiple microwave devices has been first studied by Mink in [15]. There, a large relative to the design wavelength Fabry-Perot cavity of two reflectors, plane total reflector and perforated (PRS) radiation shell, with a spherical curvature is applied. Such a large relative to wavelength $1 \mathrm{D}$ cavity is more suitable for millimeter and submillimeter (quasi-optical) antenna-oscillators. In [15], the planar array of IMPATT or Gunn-diodes is placed in a small distance from the total plane reflector.

Intensive and fruitful work on efficient $1 \mathrm{D}$ cavity power combining devices is described later on in [16-19]. However, because of the bigger diffraction loss and inner cavity field distribution change, their radiation characteristics are inferior compared to those of the corresponding passive cavity antennas.

For small and medium aperture-size microwave and long-mm-wave passive and active antennas the use of $3 \mathrm{D}$ antenna cavities has some advantages.

(i) There is no diffraction loss as in the case of the laterally open quasi-optical cavities, and thus better radiation characteristics and higher gain and efficiency are possible.

(ii) The metal closed cavity is a robust container for the solid-state electronic circuitry placed inside.

Early work on the power combining in $3 \mathrm{D}$ round cavity antenna-oscillators is found in [20]. These are X-band single-cavity and double-cavity antenna-oscillators containing Gunn-diodes integrated in half-loop feeds, where power combining efficiency is bigger than $80 \%$ and aperture radiation efficiency is $40-50 \%$ for the two-cavity and bigger than $60 \%$ for the single-cavity antenna oscillators.

In this paper an effective 3D cavity antenna-oscillator comprising an array of $2 \times 2$ MESFET oscillator elements and radiating through a rectangular slotted-array aperture is contrasted to a similar in shape and dimensions 3D passive cavity antenna.

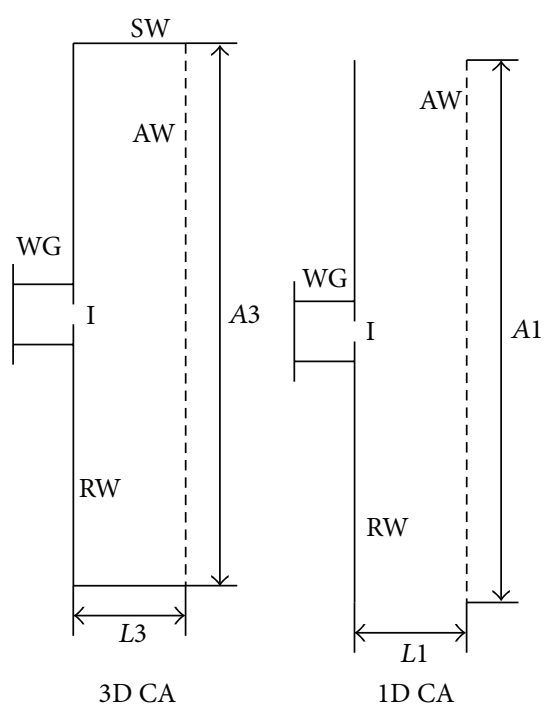

(a)

(b)

FIgURE 1: Profiles of passive cavity antennas: (a) 3D passive cavity antenna, (b) 1D passive cavity antenna.

\section{Profiles and Resonant Features of Passive and Active Cavity Antennas}

2.1. Profiles of Passive Cavity Antennas. Profile cross-sections of $3 \mathrm{D}$ and $1 \mathrm{D}$ passive cavity antennas are sketched in Figures 1(a) and 1(b), respectively. Each antenna cavity is fed in the middle of the back reflector wall (BW) by a flanged (F) hollow waveguide (WG) through an end-wall iris (I). The 3D cavity antenna (3D CA) is laterally closed by a side wall (SW), while the $1 \mathrm{D}$ cavity antenna (1D CA) is laterally open. $A 3$ and $A 1$ are the transverse profile cavity dimensions, while $L 3$ and $L 1$ are the lengths of $3 \mathrm{D}$ cavity and $1 \mathrm{D}$ cavity, correspondingly. Both cavity antennas, 3D CA and 1D CA, have partially reflecting aperture wall (AW).

2.2. Profiles of Active Cavity Antennas-Oscillators. Figures 2(a) and 2(b) illustrate the profiles of 3D and 1D cavity antennas-oscillators.

The passive cavity antennas illustrated above (Figure 1) are converted here into the active (antennas-oscillators) sketched in Figure 2. It is supposed that these antennasoscillators have the same cavity shapes and dimensions as the corresponding passive ones but are fed by inner (integrated) autonomous grid-MESFET oscillators (GOs).

2.3. Mode Spectrum Density in Lossless Cavities. In the 3D or closed cavity a number of natural field modes can be excited. Their resonant frequencies form a discrete spectrum in the cavity volume $V$ with a spectrum density $N / \Delta f$ given by the asymptotic Rayleigh-Jeans formula [21]

$$
\frac{N}{\Delta f} \approx 4 \pi \frac{V}{c^{3}} f^{2},
$$




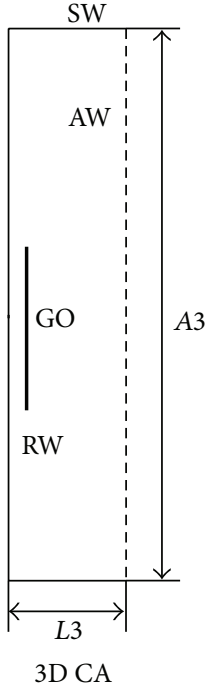

(a)

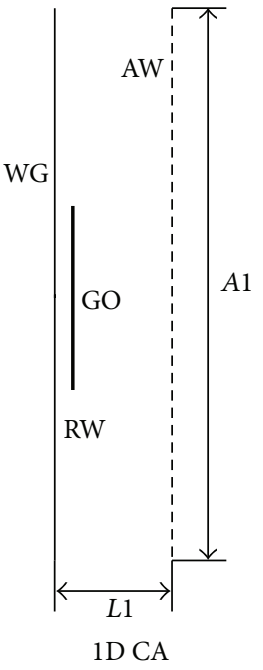

(b)
FIGURE 2: Profiles of active cavity antennas: (a) 3D cavity antennasoscillators, (b) 1D cavity antenna-oscillator.

where $N$ is the number of field modes for a unit frequency increment and $c$ is the free-space light velocity. Obviously, the mode discrete spectrum density becomes bigger with the volume and frequency increase $\left(N / \Delta f \sim V f^{2}\right)$.

In the $1 \mathrm{D}$ or Fabry-Perot cavity the spectral density does not depend on the frequency but on the cavity length $L$ only

$$
\frac{N}{\Delta f} \approx \frac{2 L}{\pi c} .
$$

Or, evidently, the resonant frequencies form an equidistant (constant-density) spectrum.

It is concluded that as a result of the lateral opening in the $1 \mathrm{D}$ cavity there are no transverse field reflections and transverse cavity modes. Thus, the latter are selectively radiated and only the axial standing-wave modes are left inside. This is the physical explanation for the frequency spectrum thinning effect.

Like the cavity resonator the passive or active cavity antenna is supposed to operate in a single-mode regime; that is, in a specific frequency band only a single characteristic mode exists. Most often this is the fundamental mode with the lowest resonant frequency, which is achieved by choosing a proper cavity size and field excitation and suppression (filtering) of unwanted modes.

\section{Passive and Active 3D Cavity Antenna Designs}

3.1. Passive Cavity Antenna Design. The passive cavity antenna (Figure 1(a)) comprises a 3D rectangular cavity. The antenna is designed for a resonant frequency of $9.0 \mathrm{GHz}$ (or resonant wavelength of $33.33 \mathrm{~mm}$ ). The cavity is excited by means of $\mathrm{TE}_{10}$-mode rectangular waveguide through an endwall capacitive iris $23 \mathrm{~mm} \times 4 \mathrm{~mm}$ in size.
Our experience with the passive cavity antennas has shown that the fundamental cavity field mode $\mathrm{TE}_{101}$ very much prevails over the nearest higher-order TE and $\mathrm{TH}$ modes if the characteristic transverse cavity size $A$ is less than $2.5-3.0 \lambda_{\mathrm{TE}_{101}}$, where $\lambda_{\mathrm{TE}_{101}} \approx \lambda_{0}$ is the cavity $\mathrm{TE}_{101}$-mode resonance frequency [2-4]. The cavity aperture wall (AW) is chosen square in shape with dimensions as follows: aperture side $A=B=80 \mathrm{~mm}$ (or $2.4 \lambda_{0}$ ) and cavity length $L=16 \mathrm{~mm}$ (or $0.48 \lambda_{0}$ ).

For a lossless and nonradiating rectangular cavity the resonance frequency of the fundamental $\mathrm{TE}_{101}$ mode is easily found by

$$
f_{101}=\frac{c}{2} \sqrt{\frac{1}{A^{2}}+\frac{1}{L^{2}}} \text {. }
$$

The corresponding cavity wavelength is

$$
\lambda_{101}=\frac{2}{f_{101}} .
$$

This passive cavity antenna radiates through a slotted (partially reflecting) aperture printed on a copper-clad dielectric substrate of thickness $1.5 \mathrm{~mm}$ and permittivity and loss tangent equal to 2.3 and 0.005 , respectively. The aperture slot array consists of $4 \times 5=20$ equal slots, with a size of $15 \mathrm{~mm} \times 2.5 \mathrm{~mm}$ each.

3.2. Cavity Antenna-Oscillator Design. The passive cavity antenna described in Section 3.1 is converted here into the cavity antenna-oscillator sketched in Figure 3. This active antenna has the cavity of the passive one but is fed by an inner grid of $2 \times 2$ MESFET transistor oscillators, similar to those described in $[22,23]$. Each MESFET-oscillator element has the configuration, dimensions, and electric data shown in Figure 3(a). The grid plate is centered on the cavity wall opposite to the slotted aperture wall (Figure 3(b)). A feedback coupling occurs between the basic cavity field mode $\mathrm{TE}_{101}$ and the oscillator array dipole elements. This phenomenon leads to an injection phase-locking and high efficient power combing in the closed cavity volume. Like the semitransparent slotted aperture plate, the MESFET grid oscillator circuit is printed on a copper-clad Teflon substrate. The detuning effect of RF induced biasing caused by the cavity field mode to the MESFET oscillator action is not considered initially. The grid-oscillator is designed for an action in free space. As expected, after its insertion in the antenna high$\mathrm{Q}$ resonant cavity, the oscillator phase noise and stability performance have been improved. Small adjustments of the carrier frequency and radiation efficiency have been done with a change of the cavity and dipoles' lengths.

The MITSUBISHI MGF 1303B MESFETs are employed in the grid oscillator. Within the $\mathrm{X}$-band each transistor provides about $16 \mathrm{~mW} \mathrm{CW}$ power.

3.3. Experimental Results for Passive and Active Cavity Antennas. Figure 4 illustrates the copolarization (solid lines) and cross-polarization (dashed lines) radiation patterns of the studied cavity antenna-oscillator measured at $8.62 \mathrm{GHz}$ in the 


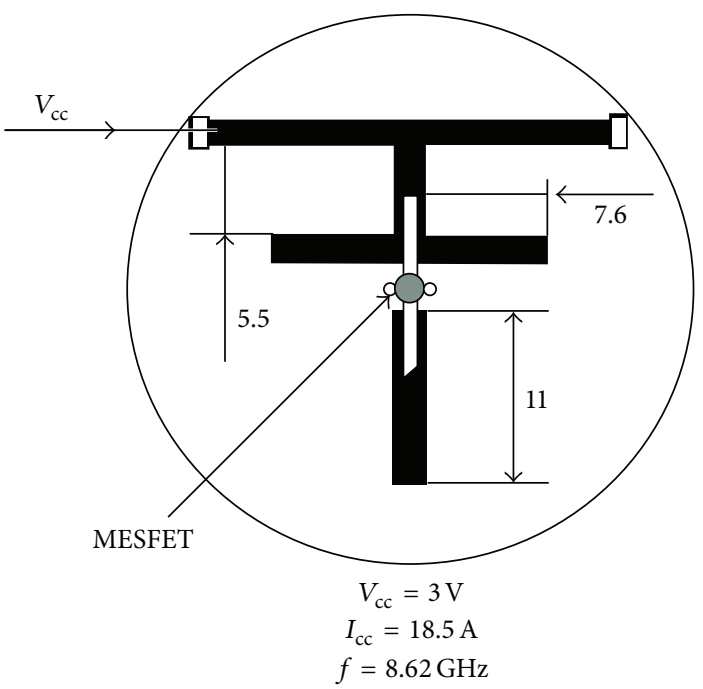

(a)

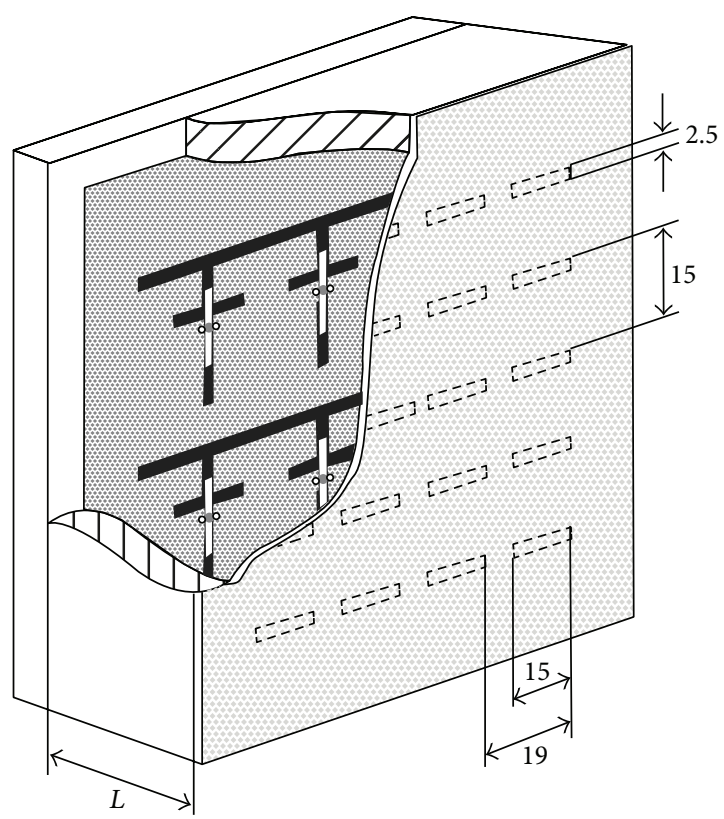

(b)

FIGURE 3: X-band cavity grid-oscillator: (a) single-oscillator schematic and design data and (b) $2 \times 2$ MESFET grid oscillator integrated into passive cavity antenna.

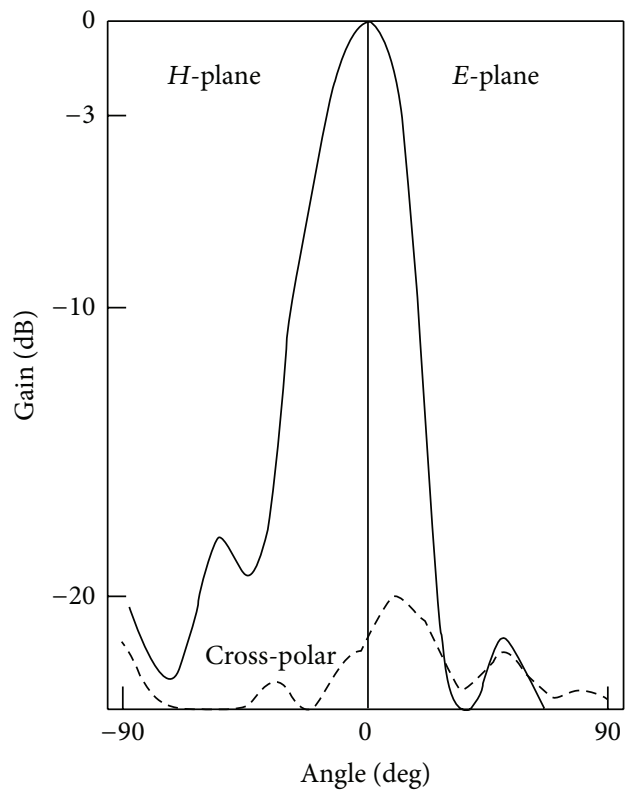

FIgURE 4: Measured copolar (solid lines) and cross-polar (dashed lines) $H$-plane (left graphs) and $E$-plane (right graphs) radiation patterns of cavity antenna-oscillator.

main antenna planes: $E$-plane (right curves) and $H$-plane (left curves). Evidently, the copolarization $E$-plane radiation pattern has narrower beamwidth and lower maximum sidelobes, while the maximum $H$-plane cross-polarization is smaller compared to the $E$-plane one.

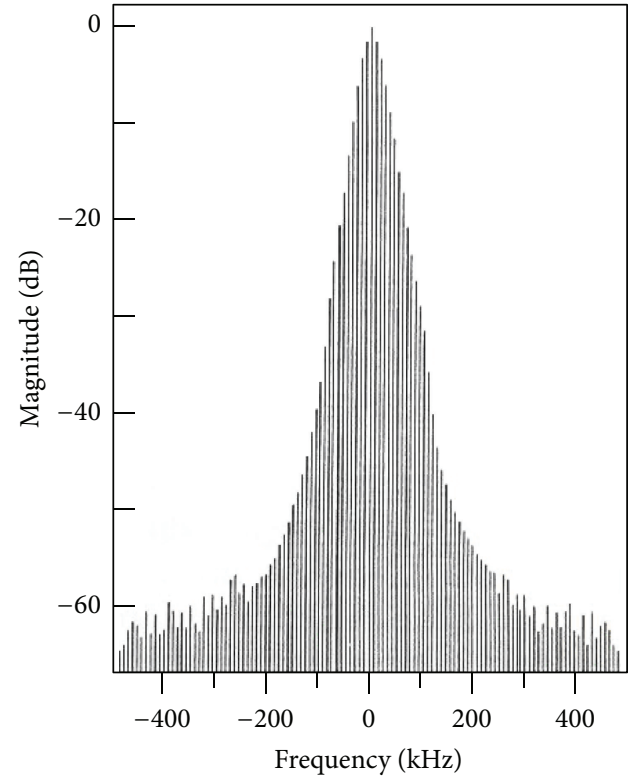

FIGURE 5: Frequency spectrum of cavity-oscillator antenna measured in antenna far-field region for carrier frequency of $8.62 \mathrm{GHz}$

The frequency spectrum of cavity antenna-oscillator measured in antenna far-field region is shown in Figure 5. The carrier oscillator frequency is $8.62 \mathrm{GHz}$ and the measured SSB spectral power density is $-82 \mathrm{dBc} / \mathrm{Hz}$ at $100 \mathrm{KHz}$ off the carrier frequency. Overall DC to RF efficiency of $26 \%$ and effective isotropic radiated power (EIRP) of $5.2 \mathrm{~W}$ are computed from the experimental data. Further increase of DC to RF 
TABLE 1: Radiation parameters of passive and active (oscillator) cavity antennas.

\begin{tabular}{lcc}
\hline Antenna parameters & Passive antenna & Active antenna \\
\hline (1) $H$-plane rad. pattern & & \\
BW, deg & 24 & 25 \\
SLL, dB & -21 & -18 \\
(2) $E$-plane rad. pattern & & \\
BW, deg & 22 & 23 \\
SLL, dB & -23 & -22 \\
(3) $f_{101}, \mathrm{GHz}$ & 8.779 & 8.623 \\
(4) $G, \mathrm{~dB}$ & 17.3 & 16.9 \\
(5) $\eta, \%$ & 78.4 & 74.8 \\
(6) $\mathrm{DC}$ to $\mathrm{RF}$ efficiency, $\%$ & - & 26.0 \\
(7) EIRP, W & & 5.2 \\
(8) SSB, dBc/Hz & - & -82.0 \\
\hline
\end{tabular}

efficiency and EIRP is possible by further diminishing of mutual coupling in the grid-dipole array and material loss in the oscillator circuitry and antenna cavity.

In Table 1 the measured radiation characteristics of the passive cavity antenna at the resonance frequency of $8.779 \mathrm{GHz}$ and active cavity antenna-oscillator at the carrier oscillator resonance frequency are contrasted. These are beamwidth BW and first sidelobe level SLL of the E-plane and $H$-plane radiation patterns, fundamental resonance frequency $f_{101}$ in $\mathrm{GHz}$, antenna directive gain $G$ in $\mathrm{dB}$, radiation aperture efficiency $\eta=4 \pi S_{a} / \lambda_{0}^{2}$, in $\%$, where $S_{a}$ is the antenna aperture surface, $D C$ to $R F$ efficiency in $\%$, EIRP in W, and SSB spectral density in $\mathrm{dBc} / \mathrm{Hz}$.

As it is chosen in the design text above, both passive and active cavity antennas have the same structures and dimensions and differ only in their field sources: narrow capacitive iris (I) fed by a rectangular waveguide in the case of passive antenna and grid-oscillator (GO) integrated within the cavity on its back cavity wall RW.

From Figures 4 and 5 and Table 1 several important comments follow.

(1) The basic radiation parameters (BW, SLL, $\eta$, etc.) of the cavity antenna-oscillator reasonably deteriorate compared to those of the original passive cavity antenna. These effects are due to the inevitable field distribution change because of the presence of the grid-oscillator circuitry.

(2) The antenna-oscillator directive gain and efficiency compared to those of the passive cavity antenna are falling down by only $0.4 \mathrm{~dB}$ and $3.6 \%$, respectively.

(3) Both passive and active 3D cavity antennas enjoy high aperture gain efficiency of about $75-80 \%$. In contrast, the $1 \mathrm{D}$ cavity antennas for the same relatively small cavity and aperture dimensions typically have about 1.5-2.0 times smaller radiation efficiency.

(4) Insertion of grid-oscillator circuitry printed on a dielectric substrate diminishes the cavity resonance frequency, from $8.779 \mathrm{GHz}$ to $8.623 \mathrm{GHz}$, but it could be corrected simply by changing slightly the cavity length $L$ and optimizing the mutual coupling between the oscillator-array dipole elements.

\section{Conclusions}

An undersized 3D cavity antenna integrated with a fourMESFET-transistor grid-oscillator power combiner is designed and studied experimentally. Very good DC to RF oscillator efficiency of $26 \%$ and effective isotropic radiated power (EIRP) of $5.2 \mathrm{~W}$ are obtained from the measured data. It is proved that the use of $3 \mathrm{D}$ antenna resonant cavity instead of $1 \mathrm{D}$ parallel-plane one in case of small and medium aperture-size and gain microwave passive and active antennas has an effective power combining action. The lack of diffraction loss due to the lateral cavity opening leads to a higher directive gain of about $17 \mathrm{~dB}$ and radiation aperture efficiency bigger than $75 \%$. In addition, the $3 \mathrm{D}$ cavity serves as a strong metal container for the solid-state oscillator circuitry inside.

\section{Conflict of Interests}

The authors declare that there is no conflict of interests regarding the publication of this paper.

\section{Acknowledgment}

The authors acknowledge the Chilean Science Agency CONICYT for the support within the Anillos Project ACT-53 and Fondecyt Project 1120714.

\section{References}

[1] S. Jacobsen, E. Andersen, and M. Gronlund, "An antenna illuminated by a cavity resonator," Proceedings of the IEEE, vol. 11, pp. 1431-1435, 1963.

[2] A. M. Jassim and H. D. Hristov, "Cavity feed technique for slot-coupled microstrip patch array antenna," IEE Proceedings: Microwaves, Antennas and Propagation, vol. 142, no. 6, pp. 452456, 1995.

[3] A. Kumar and H. D. Hristov, Microwave Cavity Antennas, Artech House, Norwood, Mass, USA, 1989.

[4] S. V. Savov and H. D. Hristov, "Cavity-backed slot array analysis," IEE Proceedings H, vol. 134, no. 3, pp. 280-284, 1987.

[5] F. J. Paoloni, "A cavity-backed resonant slot array-theory and measurement," IEEE Transactions on Antennas and Propagation, vol. 28, no. 2, pp. 259-263, 1980.

[6] G. V. Trentini, "Partially reflecting sheet arrays," IRE Transactions on Antennas and Propagation, pp. 666-670, 1956.

[7] A. Foroozesh and L. Shafai, "On the design of high-gain resonant cavity antennas using different highly-reflective frequency selective surfaces as the superstrates," in Proceedings of the IEEE International Symposium on Antennas and Propagation and CNC-USNC/URSI Radio Science Meeting (AP-S/URSI '10), July 2010.

[8] A. P. Feresidis and J. C. Vardaxoglou, "High gain planar antenna using optimised partially reflective surfaces," IEE Proceedings: Microwaves, Antennas and Propagation, vol. 148, no. 6, pp. 345350, 2001. 
[9] R. Sauleau, P. Coquet, T. Matsui, and J.-P. Daniel, "A new concept of focusing antennas using plane-parallel Fabry-Perot cavities with nonuniform mirrors," IEEE Transactions on Antennas and Propagation, vol. 51, no. 11, pp. 3171-3175, 2003.

[10] N. Guérin, S. Enoch, G. Tayeb, P. Sabouroux, P. Vincent, and H. Legay, "A metallic Fabry-Perot directive antenna," IEEE Transactions on Antennas and Propagation, vol. 54, no. 1, pp. 220-223, 2006.

[11] Y. Ge, K. P. Esselle, and T. S. Bird, "A method to design dualband, high-directivity ebg resonator antennas using singleresonant, single-layer partially reflective surfaces," Progress in Electromagnetics Research C, vol. 13, pp. 245-257, 2010.

[12] P. J. Klass, "Helicopter stabilized by microwave beam," Aviation Week and Space Technology, vol. 22, pp. 85-89, 1968.

[13] J. R. Brauer, "Rectangular beam waveguide resonator and antenna," IEEE Transactions on Antennas and Propagation, vol. 20, no. 5, pp. 595-601, 1972.

[14] K. Fujimoto, A. Henderson, K. Hirasava, and J. James, Small Antennas, Research Studies Press, 1987.

[15] J. W. Mink, "Quasi-optical power combining of solid-state millimeter-wave sources," IEEE Transactions on Microwave Theory and Techniques, vol. 34, no. 2, pp. 273-279, 1986.

[16] Z. B. Popovic, R. M. Weikle, M. Kim, and D. B. Rutledge, "A 100-MESFET planar grid oscillator," IEEE Transactions on Microwave Theory and Techniques, vol. 39, no. 2, pp. 193-200, 1991.

[17] T. Mader, S. Bundy, and Z. B. Popovic, "Quasi-optical VCOs," IEEE Transactions on Microwave Theory and Techniques, vol. 41, no. 10, pp. 1775-1781, 1993.

[18] R. A. York and R. C. Compton, "Quasi-optical power combining using mutually synchronized oscillator arrays," IEEE Transactions on Microwave Theory and Techniques, vol. 39, no. 6, pp. 1000-1009, 1991.

[19] J. C. Wiltse and J. W. Mink, "Quasi-optical power combining of solid-state sources," Microwave Journal, vol. 144, pp. 147-150, 1992.

[20] H. D. Hristov, I. P. Gatzov, J. R. Urumov, G. S. Kirov, and T. G. Ivanov, "Some experiences with resonant cavity antennasoscillators," in Proceedings of the IEEE Antennas and Propagation International Symposium, pp. 376-381, Ann Arbor, Mich, USA, July 1993.

[21] L. A. Weinstein, Open Resonators and Open Waveguides, Golem Series in Electromagnetics, Golem Press, 1969.

[22] R. A. York, R. D. Martinez, and R. C. Compton, "Active patch antenna element for array applications," Electronics Letters, vol. 26, no. 3, pp. 494-495, 1990.

[23] E. Holtzman and R. Robertson, Solid-State Microwave Power Oscillator Design, Artech House, 1992. 

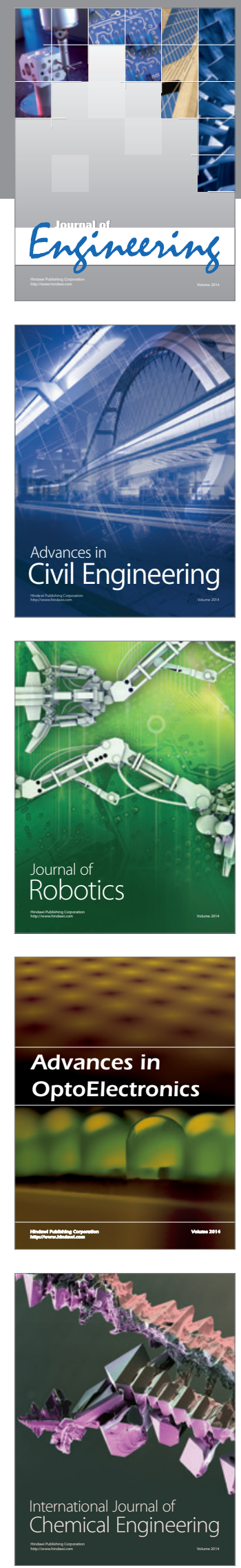

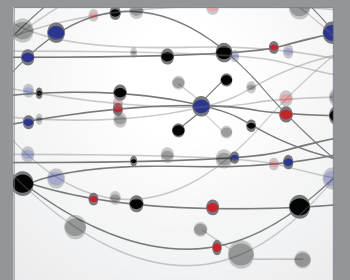

The Scientific World Journal
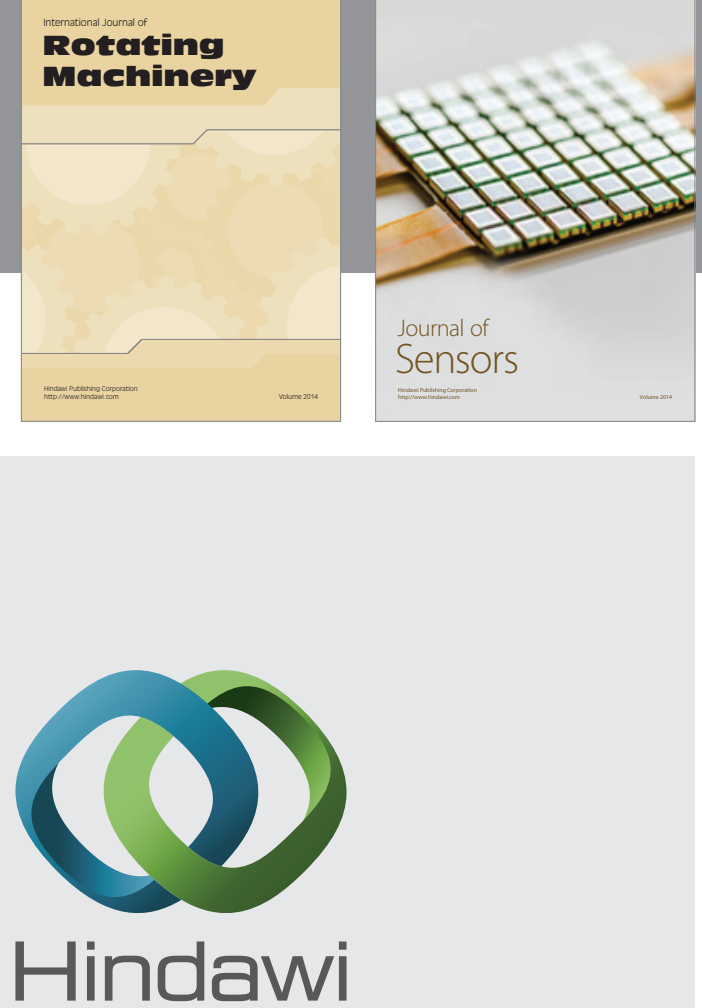

Submit your manuscripts at http://www.hindawi.com
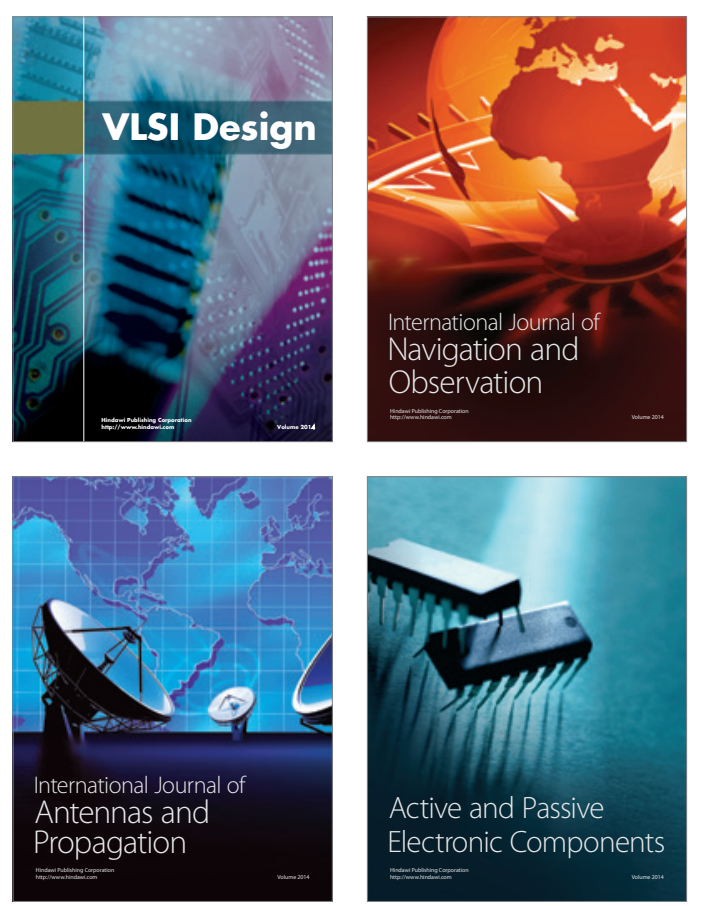
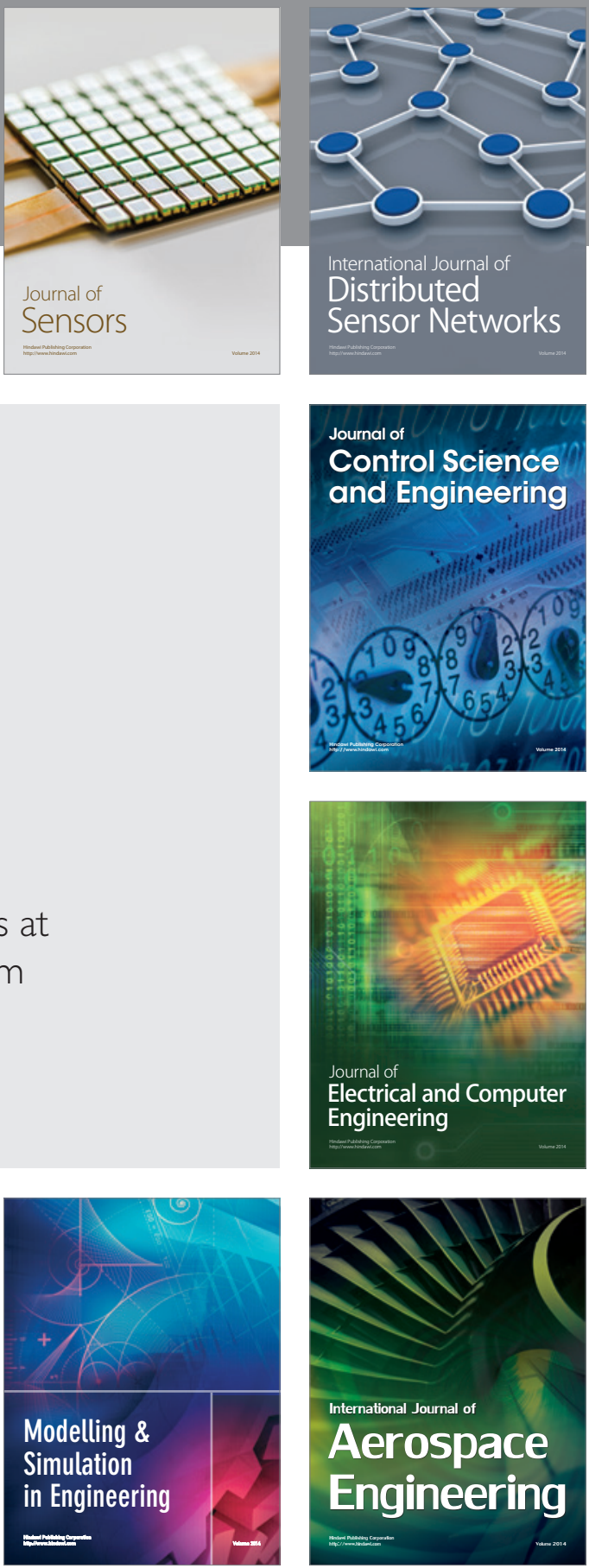

Journal of

Control Science

and Engineering
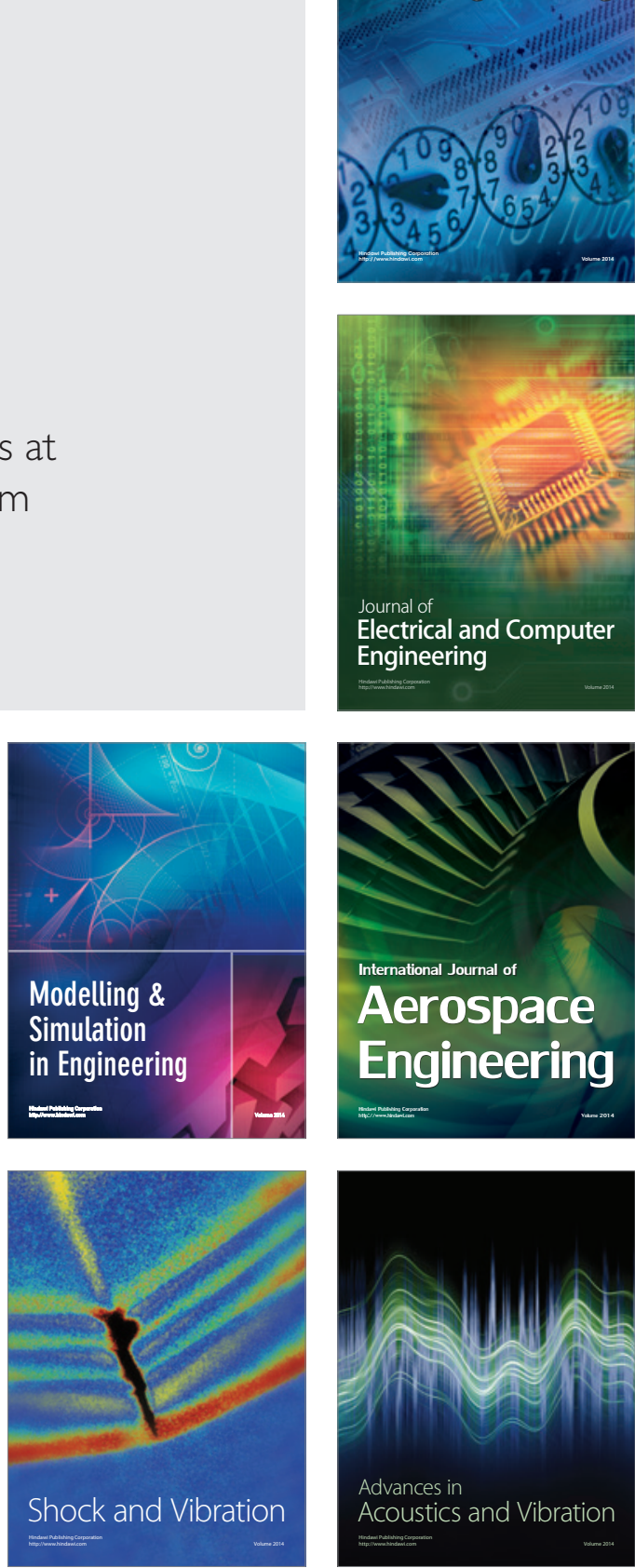